\title{
Type D personality and its impact on patients with Type 2 diabetes mellitus
}

\section{CURRENT STATUS: POSTED}

Research Square

Yi-Hsin Lin

Taiwan Adventist Hospital

Di-An Chen

Taiwan Adventist Hospital

Chemin Lin

Keelung Chang Gung Memorial Hospital of the CGMF

Hsuan Huang

Mackay Memorial Hospital

slimesin0709@gmail.comCorresponding Author

ORCiD: https://orcid.org/0000-0002-2160-3105

DOI:

10.21203/rs.2.20259/v2

SUBJECT AREAS

Psychology

KEYWORDS 


\section{Abstract}

The authors have withdrawn this preprint from Research Square 